\title{
Virus-induced Silencing of the WRKY1 Transcription Factor that Interacts with the SL1 Structure of Potato virus $X$ Leads to Higher Viral RNA Accumulation and Severe Necrotic Symptoms
}

\author{
Sang-Ho Park ${ }^{1}$ and Kook-Hyung Kim ${ }^{1,2 *}$ \\ ${ }^{I}$ Department of Agricultural Biotechnology and Plant Genomics and Breeding Institute, ${ }^{2}$ Research Institute for Agriculture and \\ Life Sciences, Seoul National University, Seoul 151-921, Korea
}

(Received on November 25, 2011; Accepted on November 27, 2011)

Potato virus $X(\mathrm{PVX})$ replication is precisely regulated by regulatory viral sequences and by viral and/or host proteins. In a previous study, we identified a 54-kDa cellular tobacco protein that bound to a region within the first 46 nucleotides (nt) of the 5 ' non-translated region (NTR) of the viral genome. Optimal binding was dependent upon the presence of an ACCA sequence at nt 10-13. To identify host factors that bind to $5^{\prime}$ NTR elements including $\mathrm{AC}$-rich sequences as well as stemloop 1 (SL1), we used northwestern blotting and matrixassisted laser desorption/ionization time-of-flight mass spectrometry for peptide mass fingerprinting. We screened several host factors that might affect $P V X$ replication and selected a candidate protein, Nicotiana tabacum WRKY transcription factor 1 (NtWRKY1). We used a Tobacco rattle virus (TRV)-based virus-induced gene silencing (VIGS) system to investigate the role of NtWRKY1 in PVX replication. Silencing of WRKY1 in Nicotiana benthamiana caused lethal apical necrosis and allowed an increase in PVX RNA accumulation. This result could reflect the balancing of $P V X$ accumulation in a systemic $N$. benthamiana host to maintain PVX survival and still produce a suitable appearance of mosaic and mottle symptoms. Our results suggest that PVX may recruit the WRKY transcription factor, which binds to the $5^{\prime}$ NTR of viral genomic RNA and acts as a key regulator of viral infection.

Keywords : NbWRKY1, SL1 RNA, RNA-host protein interaction, Potato virus $X$, replication, symptom development

The viral infection cycle involves not only viral proteins such as RNA-dependent RNA polymerase (RdRp), movement protein (MP), and capsid protein (CP) but also host proteins that interact with these viral proteins and/or viral RNA elements. For plant viruses, successful infection is

\footnotetext{
*Corresponding author.

Phone) +82-2-880-4677, FAX) +82-2-873-2317

E-mail)kookkim@snu.ac.kr
}

required for viral genome replication, cell-to-cell movement, and long-distance transport, in which diverse interactions between viral and host factors occur. On the other hand, plant defense against viruses includes the hypersensitive response (HR) and/or RNA silencing. The 5'- and 3'-nontranslated-region (NTR) sequences at the end of the viral genome contain RNA elements that are important for RNA accumulation, replication, translation, and regulation (Kim and Hemenway, 1996; Miller et al., 1998; Miller et al., 1999; Pillai-Nair et al., 2003; Sriskanda et al., 1996). The highly ordered stem-loop (SL) structures of the NTR regions, in particular, are implicated in RNA accumulation and replication through interactions with host proteins (Kwon and Kim, 2006).

Potato virus $X$ (PVX) is a flexuous rod-shaped virus in the Potexvirus genus (Alphaflexiviridae family). The PVX genome is a positive-sense single-stranded RNA encoding five open reading frames (ORFs). The $5^{\prime}$ and $3^{\prime}$ ends have a cap structure and a poly(A) tail, respectively (Huisman et al., 1988). The first ORF encodes for the viral replicase, and a region of three overlapping ORFs, called the triple-gene block (TGB), is required for virus cell-to-cell movement (Verchot-Lubicz et al., 2010; Fig. 1A). The final ORF is the viral $\mathrm{CP}$, which is necessary for virion assembly and cell-tocell movement of the virus (Cruz et al., 1998; Huisman et al., 1988; Kwon et al., 2005; Fig. 1A). The 5' region of the genomic RNA contains an AC-rich single-stranded region and two RNA SL structures, named 5' SL1 and 5' SL2 (Kim and Hemenway, 1996; Miller et al., 1998; Fig. 1B). The 5' SL1 is known as a multifunctional element involved in viral replication, cell-to-cell movement, and virion assembly (Kwon et al., 2005). Several modifications within the 5' SL1 have demonstrated that the wild-type (wt) 5' SL1 and the GAAA sequence within the tetra loop (TL) are important for plus-strand RNA accumulation (Kwon and Kim, 2006; Miller et al., 1999). Previous work has shown that the AC-rich single-stranded region and the 5' SL1 have binding activity toward host protein extracts (Kim et al., 2002; Kwon and Kim, 2006). However, identification and 
A

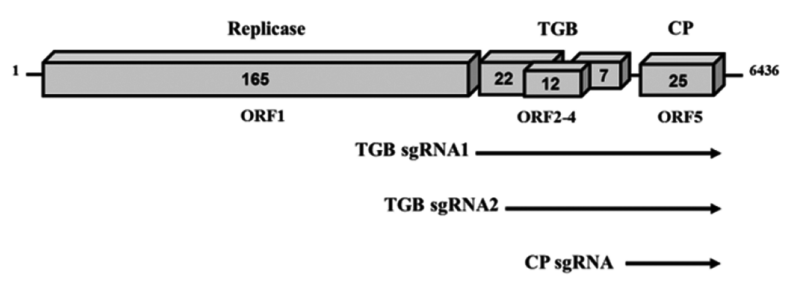

B

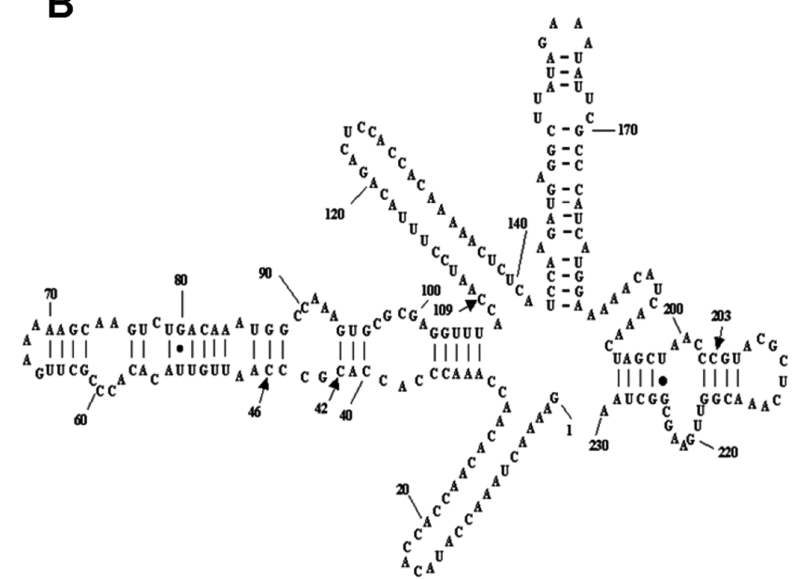

Fig. 1. Schematic diagram of the Potato virus $X$ (PVX) genome organization and predicted RNA structure adapted from Kim et al. (2002). (A) The five open reading frames are presented as boxes, and subgenomic RNAs are shown as bold arrows. (B) RNA stemloop (SL) structure of the 5' end of the PVX genome is depicted.

characterization of these host factors have not yet been determined.

Plants have evolved in an environment rich with plant pathogenic viruses and have developed a variety of defense mechanisms to protect themselves from viral infection. Perhaps the best-characterized plant antiviral mechanisms are those mediated by resistance $(\mathrm{R})$ genes. The transcriptional regulation of $\mathrm{R}$ gene expression is required for the involvement of transcription factors such as the WRKY transcription factor family (Ulker and Somssich, 2004). Genetic evidence linking a WRKY gene with resistance came from studies of the interaction of tobacco WRKY with Tobacco mosaic virus (TMV). Resistance to TMV is mediated by the tobacco resistance gene $\mathrm{N}$ and the downregulation of tobacco WRKY genes that compromise Nmediated resistance (Liu et al., 2004).

In this study, we searched host proteins involved in PVX RNA accumulation by using the 5 ' end of the genome as a probe. We used northwestern blotting and matrix-assisted laser desorption/ionization time-of-flight mass spectrometry (MALDI-TOF MS) analyses to identify host proteins that bind to the $5^{\prime}$ end of the PVX genome. We identified one candidate, the Nicotiana tabacum WRKY1 transcription factor (NtWRKY1), by peptide mass fingerprinting (MS-
Fit) and confirmed its binding with the SL1 secondary structure by gel shift assay. Virus-induced gene-silencing (VIGS) analysis was utilized to investigate the biological role of the NtWRKY1 in PVX-infected plants. The results indicate that silencing of NtWRKY1 in Nicotiana benthamiana caused plants to exhibit lethal apical necrosis by increasing PVX accumulation, suggesting that NtWRKY1 functions to regulate multiple defense response genes.

\section{Materials and Methods}

Preparation of plasmid constructs and RNA transcripts. The plasmid pMON8453 containing a PVX cDNA clone (Hemenway et al., 1990) was used to amplify each DNA fragment including a region from 1-203 nucleotides (nt) and others from 1-43 nt and the SL1 region (nt 32-106). Amplified DNA fragments were cloned into the $\mathrm{pGEM}^{\circledR}-\mathrm{T}$ Easy vector (Promega, Madison, WI, USA) generating the following plasmids: pG-203 containing nt 1-203, pG-43 containing nt 1-43, and pG-SL1 containing nt 32-106 from the 5 ' end of the PVX genome. Radiolabeled ${ }^{32} \mathrm{P}$-RNA probes were obtained by in vitro transcription in the presence of $50 \mathrm{Ci}$ of ${ }^{32} \mathrm{P}-\mathrm{CTP}(3000 \mathrm{Ci} / \mathrm{mmol}$; GE Healthcare, Piscataway, NJ, USA), $12.5 \mu \mathrm{M}$ CTP, and $500 \mu \mathrm{M}$ each of ATP, GTP, and UTP using each linearized DNA template. For the polymerase chain reaction (PCR), strand-specific primers were used as described previously (Kim et al., 2002). Each PCR product was radioactively labeled in the presence of $50 \mu \mathrm{Ci}$ of $\alpha-{ }^{32} \mathrm{P}$ CTP $(3000 \mathrm{Ci} / \mathrm{mmol}$; GE Healthcare), $5 \mathrm{mM}$ CTP, and $125 \mathrm{mM}$ each of ATP, GTP, and UTP. Labeled RNA was purified by NucAway Spin Columns (Ambion, Austin, TX, USA).

One- and two-dimensional SDS-PAGE (1-DE and 2DE). The protein pellets of S100 extracts were solubilized in $250 \mu \mathrm{l}$ of rehydration buffer containing $8 \mathrm{M}$ urea, $2 \%$ CHAPS, $0.5 \%$ IPG pH 4-7 buffer (GE healthcare), $18 \mathrm{mM}$ DTT, and bromophenol blue. Isoelectric focusing (IEF) was performed at $3500 \mathrm{~V}$ for $20 \mathrm{~h}$ at $20^{\circ} \mathrm{C}$ using IPG strips (pH 4-7 L, $13 \mathrm{~cm}$, GE Healthcare) and an Ettan IPGphor II system (GE Healthcare). Focused strips were equilibrated in $10 \mathrm{ml}$ of $6 \mathrm{M}$ Urea, $30 \%$ glycerol, $2 \%$ SDS, $125 \mathrm{mM}$ DTT, and $0.992 \%$ bromophenol blue in $50 \mathrm{mM}$ Tris-HCL with $\mathrm{pH} 8.0$, twice for $15 \mathrm{~min}$ at room temperature (RT) and then treated with $10 \%$ tricine acrylamide gel for SDSPAGE. Proteins were transferred to nitrocellulose membranes and then applied to northwestern blot analysis.

Northwestern blotting and MS-Fit analysis. S100 protein extracts were prepared from Nicotiana tabacum cv. Bright Yellow (BY2) suspension cells as described previously (Kim et al., 2002). S100 proteins and PVX CP recombinant 
protein were resolved on a $12 \%$ SDS-polyacrylamide gel and transferred to a fluoride (PVDF)-P membrane. The membrane was incubated with $6 \mathrm{M}$ guanidine hydrochloride $(\mathrm{GuHCl})$ in northwestern buffer containing $10 \mathrm{mM}$ Tris- $\mathrm{HCl}$ (pH 6.8), $25 \mathrm{mM} \mathrm{NaCl}, 1 \mathrm{mM}$ EDTA, $0.04 \%$ BSA, $0.04 \%$ Ficoll 400 , and $0.02 \%$ polyvinyl pyrrolidone40 for $30 \mathrm{~min}$ to denature proteins on the membrane, followed by washing with $6,3,1.5,0.75,0.375$, and 0.187 $\mathrm{M} \mathrm{GuHCl}$ in northwestern buffer to renature proteins. The membranes were blocked with 5\% fat-free dry milk and 20 $\mu \mathrm{g} / \mathrm{ml}$ yeast tRNA in northwestern buffer for $1 \mathrm{~h}$ at RT. After subsequent washing with northwestern buffer three times for $15 \mathrm{~min}$, membranes were air dried and exposed in a BAS imaging plate (IP) for autoradiography (Fuji film, Tokyo, Japan). The bands corresponding to the spots of the two-DE northwestern blot were excised from the gel, digested with trypsin, and analyzed by MALDI-TOF MS. The data from MALDI-TOF MS were analyzed using the Protein Prospector algorithm (http://prospector.ucsf.edu) MS-Fit with the NCBI protein database.

Construction and purification of recombinant PVX CP and NtWRKY1. The PVX CP full-length and NtWRKY1 sequences were amplified by PCR from the PVX fulllength construct pSPVXp31 (Park and Kim, 2006) and NtWRKY1 cDNA from $N$. tabacum BY2 suspension cells, respectively. PCR products were inserted into the expression vector pMAL-c2X (New England Biolabs, Ipswich, MA, USA). The Escherichia coli expression strain BL21 (DE3) was transformed with the plasmid pMAL-c2X containing PVX CP and NtWRKY1. The maltose binding protein (MBP)-PVX CP and MBP-NtWRKY1 fusion proteins were purified according to the manufacturer's instructions (New England Biolabs). The extracted protein concentrations were determined by Bradford analysis.

Electrophoretic gel mobility shift assay (EMSA). Approximately $1 \mu \mathrm{g}$ of the recombinant proteins were incubated with $\alpha-{ }^{32} \mathrm{P}$-labelled transcripts corresponding to nt 1-203, 1-43, and 32-106 of PVX RNA ( $\sim 50,000 \mathrm{cpm})$ for $10 \mathrm{~min}$ on ice with EMSA binding buffer $(20 \mathrm{mM}$ Tris$\mathrm{HCl}, \mathrm{pH}$ 7.0, 1 mM DTT, $100 \mathrm{mM} \mathrm{KCl}, 1$ mM EDTA, 100 $\mathrm{mM} \mathrm{NaCl}, 25 \mathrm{ng} / \mu \mathrm{l}$ of yeast tRNA, and $4 \mathrm{U}$ of RNase inhibitor). After the incubation, the reactions were analyzed by $5 \%$ non-denaturing polyacrylamide gel electrophoresis (PAGE) in 0.5X Tris-borate-EDTA buffer. The gels were dried, exposed to a BAS imaging plate (FUJIX Fuji Photo Film), and analyzed in a phosphorimager (Fujix BAS 2500, Fuji Film). The band intensities were analyzed with Fuji Multi Gauge v2.2 (Fuji Film) and Excel (Microsoft) software.

Subcellular localization and overexpression. The sub- cellular localization of NtWRKY1 was determined using the pSITE-2NA-NtWRKY1-GFP fusion protein and nucleus marker RFP:NbH2B as previously described (Chakrabarty et al., 2007). Onion epidermal cell layers were peeled and placed (with insides up) on Murashige and Skoog (MS) agar media. NtWRKY1-GFP and RFP:NbH2B plasmid DNA $(0.5 \mu \mathrm{g})$ were introduced into the onion cells using a PDS-He biolistic particle gun (Bio-Rad, Hercules, CA, USA). Bombardment was conducted at $10 \mathrm{~cm}$ of target distance using $0.7 \mu \mathrm{m}$ gold microcarriers under the vacuum of 28-inch Hg, helium pressure of 1100 psi (Takeuchi et al., 1992). The bombarded tissues were incubated on the MS plates for $12 \mathrm{~h}$ at $25^{\circ} \mathrm{C}$ in the dark. Samples were observed using a UV light incident fluorescence microscope (Zeiss, Axioskop, Germany). To prepare overexpression constructs of the NtWRKY1 and NtWRKY6, amplified DNA fragments were cloned into pPZP212 vector generating pPZPNtWRKY1 and pPZP-NtWRKY6. These constructs, pPZPNtWRKY1 and pPZP-NtWRKY6, were transformed into Agrobacterium tumefaciens strain GV2260 and agroinfiltrated together with a Tomato bushy stunt virus (TBSV) p19 (pPZP-p19)-transformed strain of A. tumefaciens into the leaves of $N$. benthamiana.

VIGS assay and PVX infection. TRV-induced gene silencing assays using pTRV1 or pTRV2 and its derivatives (pTRV2-PDS and pTRV2-NtWRKY1) were performed as described (Liu et al., 2002). Plants were maintained at $25^{\circ} \mathrm{C}$ in a growth chamber on a 16-h photoperiod. Ten days post infiltration (dpi) the upper non-infiltrated and carborundum-dusted leaf of the plant was infected with purified PVX by rub inoculation.

Total leaf RNA extraction. Leaves of $N$. benthamiana plants inoculated with TRV-based VIGS vector and purified PVX were harvested at $10 \mathrm{dpi}$ from upper non-inoculated leaves. Total RNA was isolated from $0.1 \mathrm{~g}$ of leaf samples with TRIzol $^{\circledR}$ reagent (Invitrogen, Carlsbad, CA, USA) according to the manufacturer's instructions.

Semi-quantitative RT-PCR. Total RNAs extracted from silenced and non-silenced $N$. benthamiana plant leaves were treated with TURBO DNA-free ${ }^{\mathrm{TM}}$ (Ambion) to remove contaminating genomic DNA. Next, $1 \mu \mathrm{g}$ of total RNA was reverse-transcribed using $\mathrm{M}-\mathrm{MuLV}$ reverse transcriptase (Promega) and either an NtWRKY1 specific 3' primer (5'TCAGTTAAGGAAAGAGCTG-3') or oligo (dT) 3' primer. Semi-quantitative RT-PCR was performed as previously described (Kim et al., 2010; Liu et al., 2002). PCR was performed for 15, 20, 27, 30, and 35 cycles. The intensities of the PCR bands were analyzed with GEL DOC 2000 and QUANTITY ONE (Bio-Rad). As for detection of PVX, the 
A
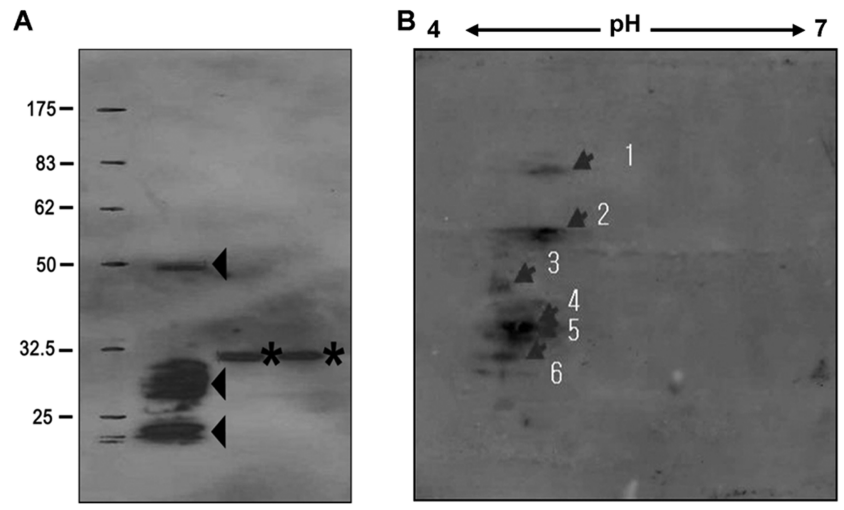

Fig. 2. Northwestern blot analysis used to identify host proteins that bind to the first 203 nucleotides (nt) of viral RNA. (A) Onedimensional electrophoresis (DE) northwestern blot analysis of S100 and recombinant PVX capsid protein (CP) with ${ }^{32} \mathrm{P}$-labled 5' 203 nt RNA. Detected host proteins are designated by arrowheads, and PVX CP are denoted by asterisks. (B) Two-DE northwestern blot analysis with S100 and recombinant PVX CP. Radiolabeled ${ }^{32} \mathrm{P} 5^{\prime} 203 \mathrm{nt}$ RNA was used as a probe. Numbers two and three were identified as NtWRKY1 and PVX CP, respectively, by MADI-TOF MS and MS-Fit.

total RNA extracted from upper non-inoculated leaves was used to detect the presence of the PVX genome by RT-PCR using PVX CP gene primers (5'-ATGTCAGCACCAGCTAGCAC-3' and 5'-TTATGGTGGTGGGAGAGTGAC-3').

\section{Results}

Host proteins that interact with the first 5' $203 \mathrm{nt}$ of the PVX RNA were identified by northwestern blot analysis.
It has been reported that RNA-protein complexes between nt 1-203 in the PVX genome (5' 203 nt) and host proteins have been formed in EMSA (Kwon et al., 2005). Interaction between the $5^{\prime}$ end of the PVX genome and host proteins was expected at various steps of the viral infection cycle due to the multiple roles of the 5 ' end. Northwestern blot analysis was used to identify host proteins from S100 extracts of $N$. tabacum BY-2 suspension cells that interact with the 5' 203 nt of viral RNA. First, 1-DE northwestern blot analysis confirmed the binding ability of the 5 ' $203 \mathrm{nt}$ RNA to host proteins. The purified CP of PVX was used as a positive control because binding ability of the $\mathrm{CP}$ to the $5^{\prime}$ SL1 in the 5 ' 203 nt has previously been reported (Kwon et al., 2005). Multiple band patterns were found, some with smaller molecular weights than $\mathrm{CP}$ and one band with a higher molecular weight, around $50 \mathrm{kDa}$ (Fig. 2A). The 1$\mathrm{DE}$ northwestern blot analysis is insufficient to effectively resolve complex protein mixtures. To determine how many distinct host proteins that interact with the 5' $203 \mathrm{nt}$ viral RNA were present in the S100 extracts, we further fractionated the S100 extracts by 2-DE SDS-PAGE. The 5' 203 nt RNA probe detected diverse proteins including the positive control, PVX CP (spot number 3), on the 2-DE northwestern blot (Fig. 2B). We excised a band from the gel corresponding to spot number 2 of the two-DE northwestern blot and analyzed it by MALDI-TOF MS. The data from MALDI-TOF MS were analyzed using MS-Fit with fixed default parameters and 50-60 kDa molecular mass, since a 54-kDa cellular protein (p54) was identified in our previous study (Kim et al., 2002). The identified proteins included a transcription factor, cyclins, a protein kinase, and
A

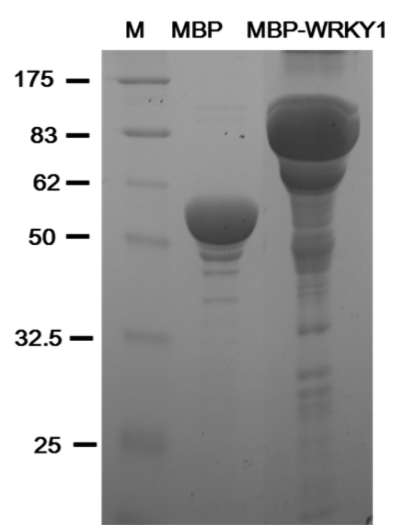

B

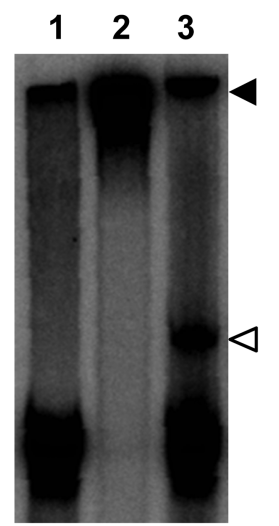

C

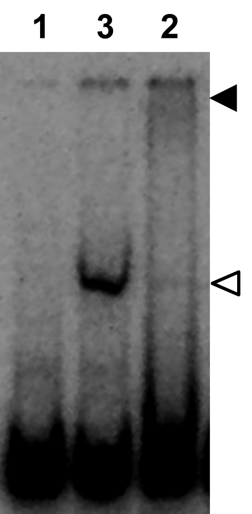

D

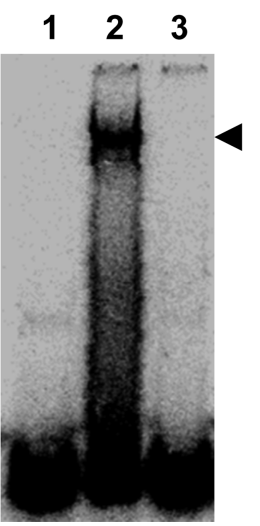

Fig. 3. Electrophoretic mobility shift assay (EMSA) of the maltose binding protein (MBP)-NtWRKY1 expressed in Escherichia coli with 5' 203 nt, 5' 43 nt, and SL1 RNA probes. (A) MBP and MBP-NtWRKY1 were purified by affinity chromatography and visualized by Coomassie-blue staining after electrophoresis through a 12\% SDS-PAGE gel. The molecular weight marker is indicated as M. (B, C, and D) The MBP or MBP-NtWRKY1 was assayed by EMSA for binding to the $\alpha_{-}{ }^{32} \mathrm{P}-\mathrm{CTP}-\mathrm{labeled} 5^{\prime} 203 \mathrm{nt}$ (B), 5 ' $43 \mathrm{nt}$, (C) and SL1 (D) RNA probes. No protein, MBP-NtWRKY1, and MBP were added (lanes 1, 2, and 3, respectively). RNA and RNA-protein complexes were separated on a $4 \%$ non-denaturing polyacrylamide gel. Closed and open arrowheads represent retarded movement by the formation of PVX 5' RNA-MBP-NtWRKY1 and PVX 5' RNA-MBP complexes, respectively. 
a dehydrogenase. The protein with the highest score, $N$. tabacum WRKY1 transcription factor (NtWRKY1), was selected and used for additional characterization. The accession number of the full-length cDNA for this protein is $\mathrm{AB} 022693$.

NtWRKY1 binds to the SL1 stem-loop structure of the 5 ' region of the PVX genome. The 5' region of the PVX genome contains an AC-rich single-stranded region and RNA stem-loop structures that are required for viral plusstrand accumulation, virus assembly, and cell-to-cell movement (Kim and Hemenway, 1996; Kwon et al., 2005; Lough et al., 2006; Miller et al., 1998; Miller et al., 1999). To confirm the in vitro binding activity of NtWRKY1 with the 5' end regions of the PVX genome, EMSA was conducted with non-secreted MBP-NtWRKY1 fusion protein (Fig. 3A). Three RNA probes, 1-203 nt, 1-43 nt, and the SL1 RNA, were prepared by in vitro transcription with $\alpha$ ${ }^{32}$ P-CTP. Distinct RNA-protein complexes exhibiting slower electrophoretic mobility were displayed when the $5^{\prime}$ 203 nt and 5' SL1 RNA probes were incubated with the MBP-NtWRKY1 fusion protein, whereas no binding was observed between MBP and either RNA probe (Fig. 3B and C). The $5^{\prime} 43$ nt probe containing the AC-rich singlestranded region (5' $43 \mathrm{nt}$ ) also bound to the MBP-NtWRKY1 fusion protein, since there was binding affinity for the MBP (Fig. 3C). Because the 5' 43 nt RNA probe possessed nonspecific binding affinity to MBP, the binding of the $5 ' 43 \mathrm{nt}$ RNA with MBP-NtWRKY1 fusion protein was not specific. These results indicated that the 5' SL1 stem loop structure in the $5^{\prime}$ region of the PVX genome could bind NtWRKY1 in vitro.

In vivo localization and overexpression of NtWRKY1. The tobacco WRKY1 transcription factor has been shown to be localized in the nucleus and to function as a trigger of HR-like cell death when the salicylic acid-induced protein kinase (SIPK) is co-expressed in a dose-dependent manner (Menke et al., 2005). To confirm these findings in our system, the NtWRKY1 coding sequence was fused to the amino termini of green fluorescent protein (GFP) in the pSITE-2NA and introduced into the pZP vector, which allows expression of NtWRKY1 within the plant without autofluorescence (Chakrabarty et al., 2007). As we expected, NtWRKY1:GFP was mainly present in the nucleus when transiently expressed with the red fluorescent protein (RFP)-fused nucleus marker, RFP:NbH2B harboring $N$. benthamiana histone $2 B$ gene, in onion epidermal cells (Fig. 4).

In contrast to previous work (Menke et al., 2005), our results show that transient overexpression of NtWRKY1 without the expression of SIPK caused a hypersensitive
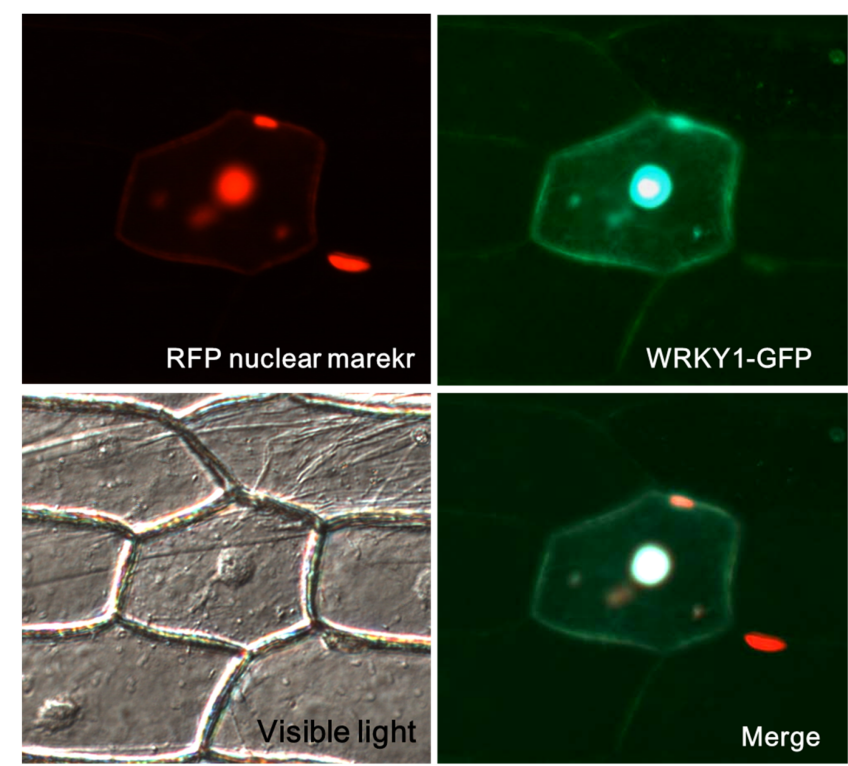

Fig. 4. Subcellular localization of NtWRKY1-GFP. NtWRKY1GFP and RFP:NbH2B (nucleus marker) were bombarded into onion epidermal cells with DNA-coated gold particles, and GFP and RFP expression was then visualized after $16 \mathrm{~h}$.
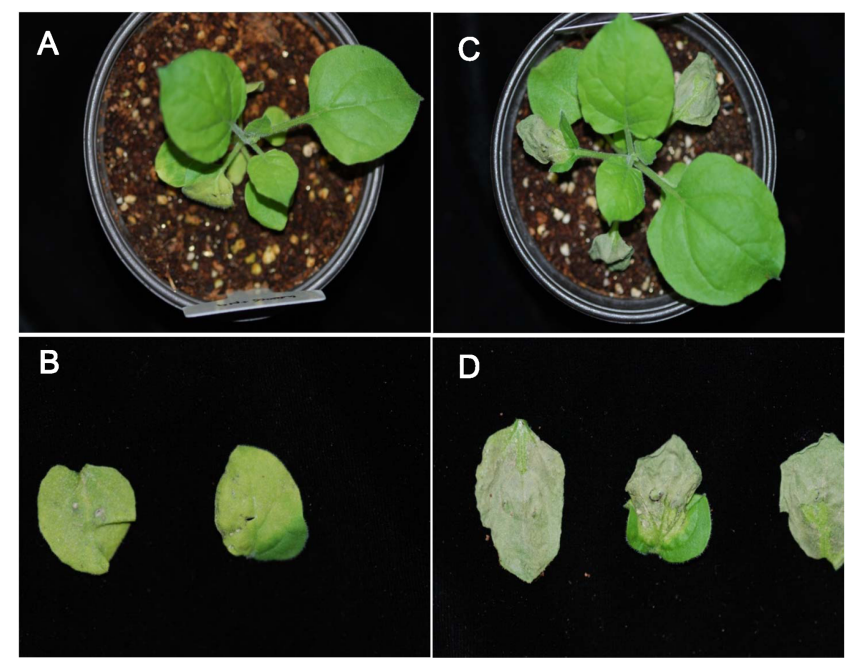

Fig. 5. Overexpression of NtWRKY1 by the silencing suppressor p19. Symptom appearances on NtWRKY6 (A and B) and NtWRKY1-overexpression (C and D) plants are shown.

response (HR)-like cell death when NtWRKY1 was coexpressed with the RNA silencing suppressor TBSV p19 in the $N$. benthamiana (Fig. 5C and D), whereas overexpression of NtWRKY6 as control did not induce cell death (Fig. 5A and B). These results suggest that NtWRKY1 activates defense genes related to HR-like cell death in the nucleus and co-expression of NtWRKY1 with either SIPK or viral RNA silencing suppressor p19 leads to more rapid cell death than the expression of NtWRKY1 alone. 
A

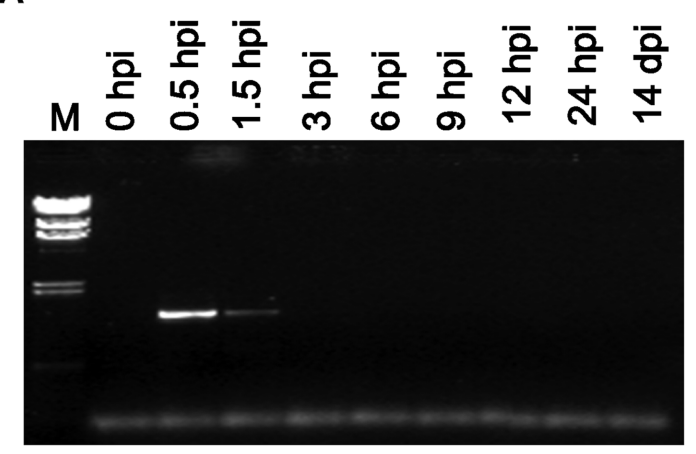

B

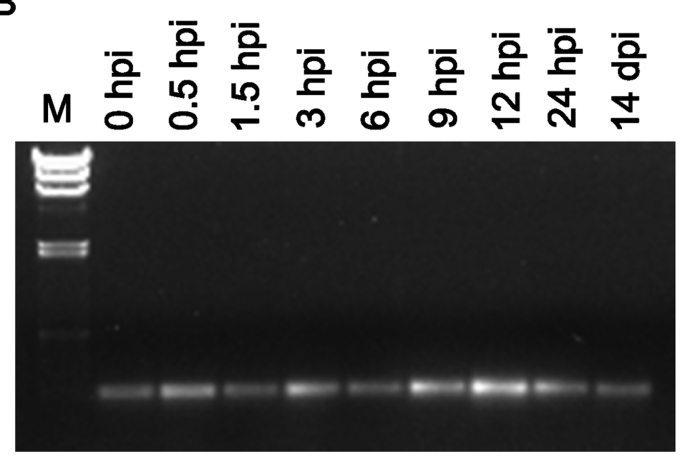

Fig. 6. Expression levels of $N t W R K Y 1$ and elongation factor 1- $\alpha$ (EF1- $\alpha$ ) genes after PVX infection. (A) NtWRKY1 mRNA levels were accumulated rapidly within 0.5 hours post-infection (hpi) and gradually decreased until $1.5 \mathrm{hpi}$. EF1- $\alpha$ was used as an endogenous gene control.

Changes in mRNA levels of NtWRKY1 during PVX infection. In another plant virus study, transcripts of the WRKY-type transcription factor TIZZ (TMV-induced leucine zipper zinc-finger) were induced in TMV-infected $n a h G$ transgenic tobacco plants (Yoda et al., 2002). To test whether PVX infection affects NtWRKY1 mRNA levels, RT-PCR was performed with total RNAs extracted from PVX-infected $N$. benthamiana at different time points. The results demonstrated that NtWRKY1 mRNA levels accumulated rapidly within 0.5 hours post-infection (hpi) (Fig. 6A). The level of NtWRKY1 mRNA reached a maximum at about 0.5 hpi and gradually decreased until 1.5 hpi (Fig. $6 \mathrm{~A}$ ), whereas very little or no difference was detected for mRNA levels of EF1-alpha (Fig. 6B). This result indicates that NtWRKY1 was induced early following PVX infection.

Effects of silencing the WRKY1 gene on PVX infection. The TRV-based VIGS vector has been used to silence genes of interest. Hence, an ortholog of the NtWRKYI gene (OtWRKY1) and a gene for endogenous phytoene desaturase $(P D S)$ were silenced in order to examine the function of the OtWRKY1 transcription factor in $N$. benthamiana (Liu et al., 2002). The PDS gene was used for assessment of the
A

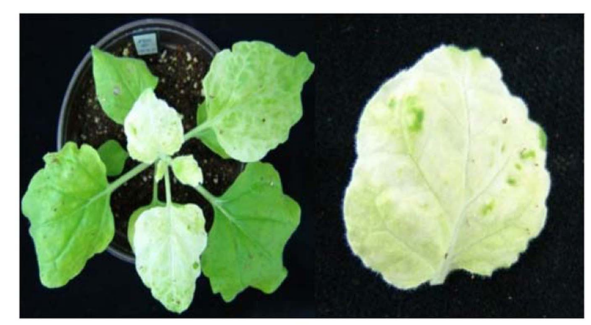

B

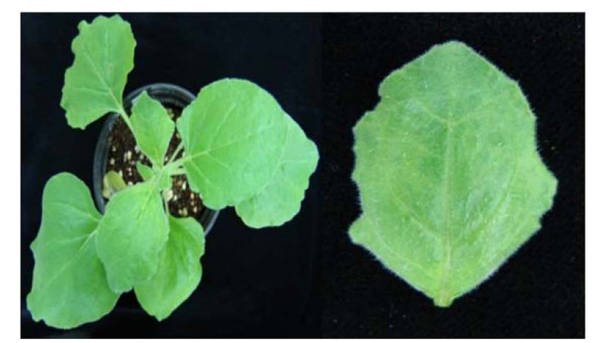

C

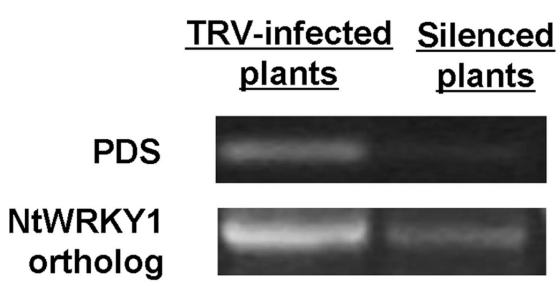

Fig. 7. Silencing of the PDS and NtWRKY1 genes. (A and B) Recombinant TRV constructs carrying either the PDS or the NtWKRY1 sequence were infiltrated into Nicotiana benthamiana plants to silence endogenous PDS and an ortholog of NtWRKY1. Whereas PDS-silenced plants had inhibited carotenoid biosynthesis, resulting in a photobleached phenotype (A), no symptom differences were detected in the ortholog of NtWRKY1silenced plants (B). The whole plant is presented on the left, and an enlarged single leaf is presented on the right. (C) RT-PCR analysis was performed on $N$. benthamiana silenced for PDS and the ortholog of NtWRKY1 to confirm silencing.

gene-silencing efficiency of our TRV VIGS system (Kumagai et al., 1995). The highest silencing efficiency of the $P D S$ gene was observed in the upper non-infiltrated leaves at 10 dpi indicating that the TRV-VIGS system could be used to induce silencing of OtWRKY1 (Fig. 7A). After 10 dpi, no visible phenotypic difference was detected in OtWRKY1silenced $N$. benthamiana when compared to that of the TRV-VIGS vector alone (Fig. 7B). Semi-quantitative RTPCR analysis suggested that mRNA levels of PDS and OtWRKY1 were reduced to $\sim 10-20 \%$ of the TRV-vector infiltrated control plant (Fig. 7C).

To verify the role of the OtWRKY1 transcription factor in PVX infection, WRKY1-silenced and TRV-VIGS vectorinfiltrated control plants were infected with PVX and monitored for 10 dpi. In TRV-VIGS vector-infiltrated control plants, PVX induced severe mosaicism with leaf curling symptoms, whereas PVX infection caused lethal apical 


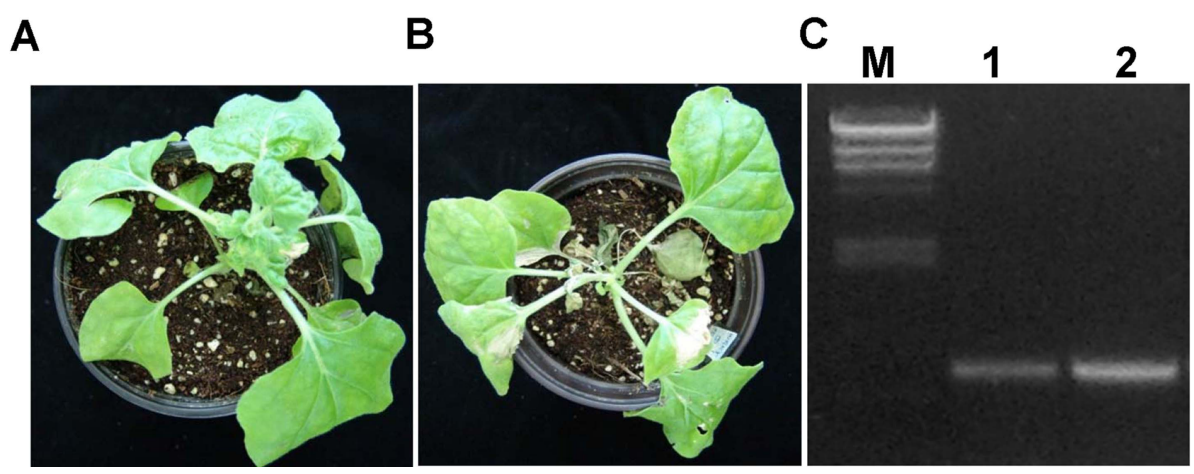

Fig. 8. Effect of $N t W R K Y 1$ silencing in $N$. benthamiana on symptom development and viral RNA accumulation. (A and B) Symptom development was observed at 10 dpi after PVX infection. Symptoms caused by PVX on Tobacco rattle virus (TRV) alone infected (A) and NtWRKY1 silenced (B) plants. (C) Viral RNA accumulation analyzed by semi-quantitative RT-PCR and 20 cycles of reactions were represented. Total RNAs were extracted from TRV alone infected (lane 1) and NtWRKY1 silenced (lane 2) plants. The molecular weight markers are indicated as $\mathrm{M}$.

necrosis (Fig. 8A and B). The levels of PVX RNA accumulation in the upper non-infiltrated leaf were analyzed by semi-quantitative RT-PCR analysis. The result indicated that silencing of OtWRKY1 increased the levels of PVX RNA accumulation compared to the non-silenced plant (Fig. 8C). The results suggest that OtWRKY1 might be implicated in balanced accumulation of the PVX genome and maintaining mosaic symptoms in $N$. benthamiana.

\section{Discussion}

We used 2-DE northwestern blot analysis to identify host proteins that bound to the first $203 \mathrm{nt}$ of the PVX genome. It has been reported that the $5^{\prime}$ end of the PVX genomic RNA is important for accumulation of genomic RNAs and sgRNAs, cell-to-cell movement, and virion assembly (Kim and Hemenway, 1996; Kwon et al., 2005; Lough et al., 2006; Miller et al., 1998; Miller et al., 1999). The fact that host proteins bound to the 5 ' end of PVX indicates that viral RNA undergoes multiple interactions with host factors and that these interactions are essential for the PVX infection cycle. Among those host proteins, the NtWRKY1 transcription factor was selected for further investigation. EMSA using the recombinant MBP-NtWRKY1 fusion protein demonstrated that the $5^{\prime}$ SL1 element, in the $5^{\prime}$ end of the PVX genome, is the direct binding partner for NtWRKY1.

WRKY transcription factors are involved in the regulation of plant defense gene expression against diverse biotic and abiotic stresses (Pandey and Somssich, 2009). The resistance response of plants is required for large-scale transcriptional reprogramming by various transcription factor families such as the WRKY genes (Eulgem, 2005; Naoumkina et al., 2008; Ryu et al., 2006). Nuclear localization was predicted for WRKY proteins and has been demonstrated for the WRKY transcription factors of parsley and tobacco (Eulgem et al., 1999; Hara et al., 2000). We also showed that the predominant nuclear localization of NtWRKY1 was confirmed in transient expression assays by particle bombardment in onion epidermal cells. Whereas DNA viruses and retroviruses are accessible to proteins that are resident in the nucleus, the site of viral-genome replication of RNA viruses is usually the cytoplasm. Therefore, the rationale for employment of transcription factors in positivestranded RNA viruses such as PVX is not immediately understood. Viruses have evolved a variety of mechanisms to overcome host defenses. This raises the possibility that transcription factors are required for limiting the host resistance response and reprogramming metabolic functions of the host in order to obtain a more favorable environment for viral multiplication. Furthermore, not all of the NtWRKY1 protein resides in the nucleus of the onion cell, which means that this protein can traffic from the nucleus to the cytoplasm. In our study, expression of NtWRKY1 was activated rapidly, within $30 \mathrm{~min}$ of PVX infection, and then gradually decreased, suggesting that NtWRKY1 residence in the nucleus is time- and/or condition-dependent. In Arabidopsis thaliana, nearly 1 out of 4 of the 72 AtWRKY proteins were transcriptionally upregulated within $2 \mathrm{~h}$ of pathogen infection or salicylic acid (SA) treatment, although almost $70 \%$ of the AtWRKY genes were differentially regulated (Dong et al., 2003; Kalde et al., 2003).

Previous studies have shown that overexpression of SIPK-triggered cell death and transient expression of tobacco WRKY1 in the presence of SIPK induced more rapid cell death than the expression of SIPK alone (Menke et al., 2005; Zhang and Liu, 2001). This suggested that WRKY1 may be a component of the signaling cascade downstream of SIPK involved in HR-like cell death (Menke et al., 2005). Cell death in the $N$. benthamiana was observed 
only after 1 week with transiently expressed NtWRKY1 alone. However, overexpression of NtWRKY1 in combination with the silencing suppressor $\mathrm{p} 19$ accelerated cell death of the plant suggesting that NtWRKY1 may activate and regulate defense response genes related to HR-like cell death. We suspect that plants precisely regulate the expression of NtWKRY1 to prevent themselves from the prolonged expression of NtWRKY1. The function of NtWRKY1 was further investigated using the TRV-based VIGS system. Our silencing results show that PVX infection induced very severe lethal apical necrotic symptoms in NtWRKY1-silenced plants, in which the levels of PVX RNA accumulation were increased compared to control plants. Together with overexpression results, this suggests that NtWRKY1 plays an important role in the control of PVX genome accumulation and maintaining mosaic symptoms in $N$. benthamiana.

In summary, our findings demonstrate the multifunctional nature of the 5 ' end of the PVX genome, specifically the SL1, which can interact with host proteins and maintain the correct balance of viral accumulation during PVX infection by interacting with WRKY1. This may be one of multiple strategies of plant viruses for survival in the systemic host, $N$. benthamiana.

\section{Acknowledgements}

This research was supported in part by grants from the Next-Generation BioGreen 21 Program (No. PJ00819801), Rural Development Administration; the National Research Foundation grant (No. 20110012328) funded by the Ministry of Education, Science, and Technology (MEST); the Vegetable Breeding Research Center (No. 710001-03) through Agriculture Research Center program from the Ministry for Food, Agriculture, Forestry and Fisheries, Republic of Korea to KHK. SHP was supported by a graduate fellowship from the MEST through the Brain Korea 21 Project.

\section{References}

Chakrabarty, R., Banerjee, R., Chung, S. M., Farman, M., Citovsky, V., Hogenhout, S. A., Tzfira, T. and Goodin, M. 2007. Psite vectors for stable integration or transient expression of autofluorescent protein fusions in plants: Probing Nicotiana benthamiana-virus interactions. Mol. Plant-Microbe Interact. 20:740-750.

Cruz, S. S., Roberts, A. G., Prior, D. A., Chapman, S. and Oparka, K. J. 1998. Cell-to-cell and phloem-mediated transport of potato virus x. The role of virions. Plant Cell 10:495-510.

Dong, J., Chen, C. and Chen, Z. 2003. Expression profiles of the arabidopsis $W R K Y$ gene superfamily during plant defense response. Plant Mol. Biol. 51:21-37.

Eulgem, T. 2005. Regulation of the arabidopsis defense transcriptome. Trends Plant Sci. 10:71-78.

Eulgem, T., Rushton, P. J., Schmelzer, E., Hahlbrock, K. and Somssich, I. E. 1999. Early nuclear events in plant defence signalling: Rapid gene activation by wrky transcription factors. EMBO J. 18:4689-4699.

Hara, K., Yagi, M., Kusano, T. and Sano, H. 2000. Rapid systemic accumulation of transcripts encoding a tobacco WRKY transcription factor upon wounding. Mol. Gen. Genet. 263:30-37.

Hemenway, C., Weiss, J., O'Connell, K. and Tumer, N. E. 1990. Characterization of infectious transcripts from a potato virus $\mathrm{X}$ cDNA clone. Virology 175:365-371.

Huisman, M. J., Linthorst, H. J., Bol, J. F. and Cornelissen, J. C. 1988. The complete nucleotide sequence of potato virus $\mathrm{X}$ and its homologies at the amino acid level with various plusstranded rna viruses. J. Gen. Virol. 69:1789-1798.

Kalde, M., Barth, M., Somssich, I. E. and Lippok, B. 2003. Members of the arabidopsis WRKY group III transcription factors are part of different plant defense signaling pathways. Mol. Plant-Microbe Interact. 16:295-305.

Kim, K.-H. and Hemenway, C. 1996. The 5' nontranslated region of potato virus $\mathrm{x}$ rna affects both genomic and subgenomic ma synthesis. J. Virol. 70:5533-5540.

Kim, K.-H., Kwon, S.-J. and Hemenway, C. 2002. Cellular protein binds to sequences near the 5 ' terminus of Potato virus $X$ rna that are important for virus replication. Virology 301:305312.

Kim, M.-K., Kwak, H.-R., Jeong, S.-G., Ko, S.-J., Lee, S.-H., Kim, J.-S., Kim, K.-H., Choi, J.-K., Choi, H.-S. and Cha, B.-J. 2010. Characteristics of Cucumber mosaic virus infecting zucchini in Korea. Plant Pathol. J. 26:139-148.

Kumagai, M. H., J. Donson, G. della-Cioppa, D. Harvey, K. Hanley and Grill, L. K. 1995. Cytoplasmic inhibition of carotenoid biosynthesis with virus-derived ma. Proc. Natl. Acad. Sci. USA 92:1679-1683.

Kwon, S.-J. and Kim, K.-H. 2006. The SL1 stem-loop structure at the 5'-end of Potato virus $X$ RNA is required for efficient binding to host proteins and for viral infectivity. Mol. Cells 21:6375.

Kwon, S.-J., Park, M.-R., Kim, K.-W., Plante, C.A., Hemenway, C. L. and Kim, K.-H. 2005. Cis-acting sequences required for coat protein binding and in vitro assembly of Potato virus $X$. Virology 334:83-97.

Liu, Y., Schiff, M. and Dinesh-Kumar, S. P. 2004. Involvement of Mek1 MAPKK, Ntf6 MAPK, WRKY/MYB transcription factors, Coil and Ctr1 in N-mediated resistance to Tobacco mosaic virus. Plant J. 38:800-809.

Liu, Y., Schiff, M., Marathe, R. and Dinesh-Kumar, S. P. 2002. Tobacco Rarl, Eds1 and Nprl/Niml like genes are required for $\mathrm{N}$-mediated resistance to Tobacco mosaic virus. Plant $J$. 30:415-429.

Lough, T. J., Lee, R. H., Emerson, S. J., Forster, R. L. and Lucas, W. J. 2006. Functional analysis of the 5 ' untranslated region of potexvirusRNA reveals a role in viral replication and cell-tocell movement. Virology 351:455-465. 
Menke, F. L., Kang, H. G., Chen, Z., Park, J. M., Kumar, D. and Klessig, D. F. 2005. Tobacco transcription factor WRKY1 is phosphorylated by the MAP kinase SIPK and mediates HRlike cell death in tobacco. Mol. Plant-Microbe Interact. 18: 1027-1034.

Miller, E. D., Kim, K.-H. and Hemenway, C. 1999. Restoration of a stem-loop structure required for potato virus X RNA accumulation indicates selection for a mismatch and a GNRA tetraloop. Virology 260:342-353.

Miller, E. D., Plante, C. A., Kim, K.-H., Brown, J. W. and Hemenway, C. 1998. Stem-loop structure in the 5 ' region of potato virus $\mathrm{X}$ genome required for plus-strand rna accumulation. $J$. Mol. Biol. 284:591-608.

Naoumkina, M. A., He, X. and Dixon, R. A. 2008. Elicitorinduced transcription factors for metabolic reprogramming of secondary metabolism in Medicago truncatula. BMC Plant Biol. 8:132.

Pandey, S. P. and Somssich, I. E. 2009. The role of WRKY transcription factors in plant immunity. Plant Physiol. 150:16481655.

Park, S.-H. and Kim, K.-H. 2006. Agroinfiltration-based Potato virus $x$ replicons to dissect the requirements of viral infection. Plant Pathol. J. 22:386-390.

Pillai-Nair, N., Kim, K.-H. and Hemenway, C. 2003. Cis-acting regulatory elements in the Potato virus $X 3^{\prime}$ non-translated region differentially affect minus-strand and plus-strand rna accumulation. J. Mol. Biol. 326:701-720.

Ryu, H. S., Han, M., Lee, S. K., Cho, J. I., Ryoo, N., Heu, S., Lee,
Y. H., Bhoo, S. H., Wang, G. L., Hahn, T. R. and Jeon, J. S. 2006. A comprehensive expression analysis of the WRKY gene superfamily in rice plants during defense response. Plant Cell Rep. 25:836-847.

Sriskanda, V. S., Pruss, G., Ge, X. and Vance, V. B. 1996. An eight-nucleotide sequence in the Potato virus $X 3^{\prime}$ untranslated region is required for both host protein binding and viral multiplication. J. Virol. 70:5266-5271.

Takeuchi, Y., Dotson, M. and Keen, N. T. 1992. Plant transformation: A simple particle bombardment device based on flowing helium. Plant Mol. Biol. 18:835-839.

Ulker, B. and Somssich, I. E. 2004. WRKY transcription factors: From DNA binding towards biological function. Curr. Opin. Plant Biol. 7:491-498.

Verchot-Lubicz, J., L. Torrance, A. G. Solovyev, S. Y. Morozov, A. O. Jackson and D. Gilmer, 2010. Varied movement strategies employed by triple gene block-encoding viruses. Mol. Plant-Microbe Interact. 23:1231-1247.

Yoda, H., Ogawa, M., Yamaguchi, Y., Koizumi, N., Kusano, T. and Sano, H. 2002. Identification of early-responsive genes associated with the hypersensitive response to Tobacco mosaic virus and characterization of a wrky-type transcription factor in tobacco plants. Mol. Genet. Genomics 267:154-161.

Zhang, S. and Liu, Y. 2001. Activation of salicylic acid-induced protein kinase, a mitogen-activated protein kinase, induces multiple defense responses in tobacco. Plant Cell 13:18771889. 